\title{
Development of a novel fully passive treadmill training paradigm for lower limb therapeutic intervention
}

\author{
M. Saiful Huq ${ }^{\mathrm{a}, *}$ and M.O. Tokhi ${ }^{\mathrm{b}}$ \\ ${ }^{a}$ Department of Mechanical and Aerospace Engineering, Carleton University, Ottawa, ON, Canada \\ ${ }^{\mathrm{b}}$ Department of Automatic Control and Systems Engineering, University of Sheffield, Sheffield, UK
}

\begin{abstract}
A simulation based study of a completely new form of body-weight supported treadmill training (BWSTT) technique which is fully passive in nature is presented in this paper. The approach does not require any powered means at the lower limbs and is implemented using a combination of coordinated joint locking/unlocking and flexible torque transfer mechanisms. The hip extension pertaining to the stance phase of the gait cycle is achieved through the stance foot being literally dragged by the treadmill belt while the required manoeuvring of the trunk is expected to be accomplished by the voluntary arm-support from the subject. The swing phase, on the other hand, is initiated through appropriately coupling the swing knee with the contralateral extending hip and eventually achieve full knee extension through switching the treadmill speed to a lower value. Considering adequate support from the able arms, the process effectively turns the frictional force at the foot-treadmill belt interface into an agent causing the required whole body mechanical energy fluctuation during the gait cycle.

The simulation platform consists of a dynamic planer (sagittal) full body humanoid model along with the treadmill model developed within a CAD based software environment interfaced with passive viscoelastic joint properties implemented in Simulink. The voluntary upper body effort as well as control of the gait cycle are also developed within MATLAB/Simulink environment. The gait cycle generated using the new concept is thoroughly investigated through this simulation study.
\end{abstract}

Keywords: Rehabilitation robotics, body-weight supported treadmill training (BWSTT), spinal cord injury (SCI), gait-cycle, control

\section{Introduction}

Body-weight supported treadmill training (BWSTT) has been found to elicit electromyographic (EMG) activity in lower extremity muscles that have little or no voluntary movement leading to a recovery of the over-ground walking ability in some people. Step like EMG activity has also been observed in subjects with a complete spinal cord injury (SCI) during BWSTT [1]. Distinct fundamental studies have revealed that locomotor training on treadmill

*Corresponding author: M. Saiful Huq, Department of Mechanical and Aerospace Engineering, Carleton University, Ottawa, ON, Canada. E-mail: mshuq@ @onnect.carleton.ca. and/or overground and balance are task-oriented [2]. Manually assisted treadmill training has been matured over more than ten years as a regular therapy for patients with SCI [3-5] as well as stroke [6-8]. Specific attributes of the outcomes include improve of balance, lower limb motor recovery and walking speed [7, 9], endurance [6] and other important gait characteristics such as symmetry, stride length, and double stance time $[7,10]$.

Despite its proven efficacy in gait training, the major limitation of the traditional treadmill therapy as a daily routine is the effort required by two or even three therapists in assisting the gait of severely affected subjects, setting the paralysed/paretic limb and controlling the trunk movement. This is laborious and therefore poses 
a limitation on the training duration. Furthermore, the therapist often needs to be in an ergonomically unfavourable seating posture while assisting the training, rendering it to strenuous work [11]. Although the use of body-weight support (BWS) in conjunction with a treadmill offers increased safety for the subject undergoing gait training and frees therapists from supporting the subject, considerable effort may be required from the therapists to guide the movement of the subject's lower limbs [12].

The use of functional electrical stimulation (FES) to elicit stepping and to assist with stance phase support is already a well known approach of reducing physical demand on the therapist. Hesse et al. [13] investigated the use of multichannel electric stimulation in combination with treadmill training and BWS in 11 non-ambulatory individuals with chronic hemiparesis. The approach was found to be more effective than standard physiotherapy in terms of restoration of gait function. A later study by Field-Fote [14] applied this technique to subjects with chronic incomplete SCI with positive outcome of increased walking speed and lower-extremity muscle strength. The study by Postans et al. [15] on 14 acute SCI patients determined the efficacy of the combination of FES with BWSTT in the rehabilitation of the subjects and compared the effect with standard physiotherapy, with the former found to have a positive effect on overground gait parameters which could potentially accelerate gait training in subjects with incomplete SCI.

Robotic assistance in the rehabilitation has been introduced since the 1990s [16]. In principle, this approach supports subject's leg movement by robotic device attached to the legs or feet, significantly reducing the load on therapists. The electromechanical gait trainer (GT-I) designed by Hesse and colleagues $[12,17]$ was motivated towards alleviating therapist's time and effort. The patients are supported in a harness and stand with their feet on motor driven foot-plates whose movement simulates the stance and swing phases in a physiological manner. In a multicentre trial with the GT-I on 120 acute non-ambulatory stroke patients, the experimental group was found to score significantly better compared to control group in all variables, viz. gait ability, the maximum gait distance and the competence in daily activities [18]. The Lokomat is a motorized four degrees-of-freedom (DoF) robotic exoskeleton worn by the patient during weight-supported stepping on a treadmill [19, 20]. It drives hip and knee (flexion/extension) through a gait like pattern using precision ball screws connected to DC motors. Preliminary results have indicated that training with Lokomat improves spatio-temporal characteristics of gait as well as temporal patterns of EMG activity [21]. ARTHuR [22] and PAM [23] are highly backdrivable robotic devices for measuring and manipulating stepping and pelvic motion respectively. Based on the principle of 'programmable footplates' the HapticWalker, a newer design from GT-I group uses linear and rotary motors to control the position of two footplates, simulating the ground beneath a subject [24].

MIT Skywalker [25] is a distinctly novel device in this arena primarily for the stroke patients. MIT Skywalker resorts to purely passive walking rather than the tradition kinematic-based approach used in all the previous robotic rehabilitation devices and hence claims to be more 'ecological', especially facilitating the 'heel strike'. Similar in essence to the passive walkers [26], MIT Skywalker makes use of the down slope for swing clearance as well as propels the leg forward (through natural dynamics) by lowering one end the walking surface only for the required leg for an appropriate part of the gait cycle. This is brought about by a split treadmill combined with a custom designed cam system to actuate the walking surface down and back to horizontal. The system is in its initial development stage and hence lacks any result based on stroke patients as yet.

The use of passive dynamics to assist part of BWSTT gait cycle is not an entirely new concept. Apart from MIT Skywalker [25], MacKnight et al. [27] presented a study to show that using inclined treadmill would assist the stance leg to achieve orientation that would facilitate FES supported locomotion without intrinsic hip flexion moment. Also it is a common practice in therapist-assisted BWSTT to increase the hip extension by treadmill [28], which in turn induces reflex hip extension and thus initiates swing phase of walking [29]. These and similar other approaches use the passive mechanism only as a part of the whole gait cycle and hence a fully passive mechanism of BWSTT on a conventional level treadmill has not been reported in the literature do date. A fully passive therapeutic treadmill training paradigm that is expected to possess simplicity due to its passive nature while requiring minimal therapist's supervision is proposed and simulated in this work. The methodology pertaining to the basic principles of the proposed approach and the development of the simulation platform is elaborated in the following Section. Next, the simulation results are presented in the Results Section, followed by a discussion 


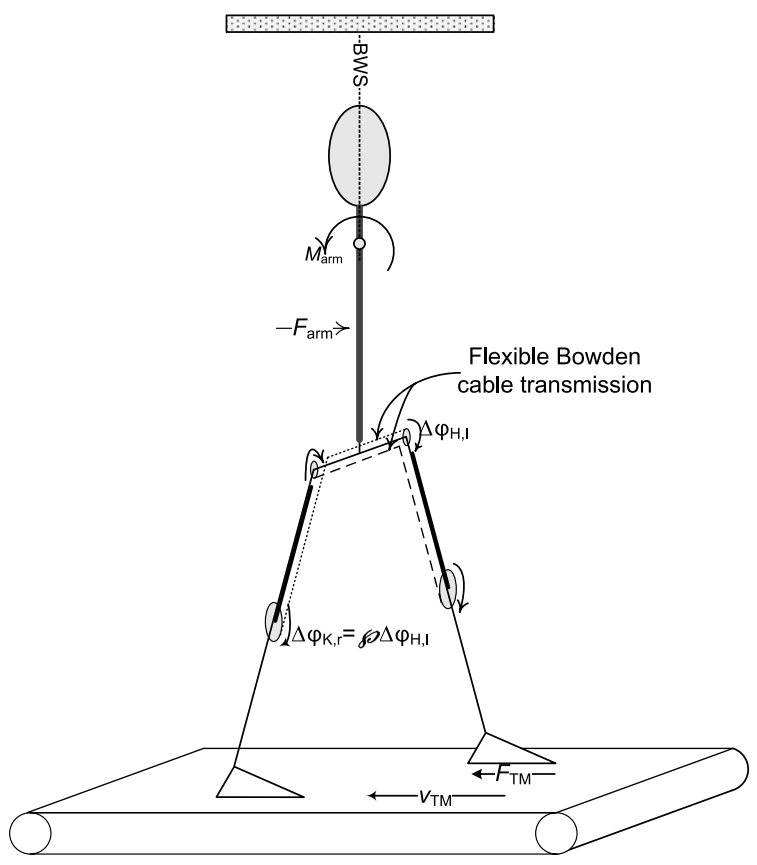

Fig. 1. Basic schematic of the proposed fully passive BWSTT with arm-support.

on the findings. The paper concludes with some final remarks stated in the Conclusions Section.

\section{Methods}

The proposed method of fully passive gait cycle generation can potentially be implemented in the form of a wearable hip-knee-ankle-foot orthosis (HKAFO) with controllable on/off brake at the knee and flexible torque transfer mechanism between the contralateral hip and knee mounted on it. Somewhat similar to approach taken by [27], the extension movement at the stance hip joint is achieved by a passive backward drag of the stance foot by the treadmill belt extending the hip joint while the subject's upper body effort (represented as $M_{\text {arm }}$ and $F_{\text {arm }}$ in Fig. 1$)$ is expected to maintain the trunk upright and in-place along the treadmill belt, thereby assisting the hip extension process. The contralateral knee joint flexion is achieved simultaneously via a flexible torque transfer mechanism acting between the extending hip and the contralateral flexing knee. The knee is then released free to gain extension movement under the influence of the gravity once desired flexion peak is achieved. The rest of the swing phase is brought about by switching the treadmill speed to a lower value, which results in some kinetic energy to be dynamically transferred to the swinging shank and hence produce full knee extension. This eventually is followed by a passive heel strike. Being fully passive in nature, the generated gait cycle is mostly determined by the passive dynamics of the lower limb segments and is associated with natural gait-like swing with ground clearance as well as near natural heel strike, events usually absent in the robotic gait trainers.

Considering the utter importance of the required minimum frictional coefficient between the foot and treadmill belt for such mechanism to take place, a detailed calculation based on first principles was carried out in [30] to establish the fundamental feasibility of the method. The value of the required frictional coefficient was found to be well within the practical limits. A detailed account of the various key elements of the proposed technique followed by descriptions of various components of the simulation platform is presented below.

\subsection{Passive swing phase generation}

While the extension movement of the stance hip is achieved through a passive drag of the foot, following steps account for the simultaneous swing phase generation on the contralateral leg (see Fig. 1):

i. The hip joint of the stance leg be coupled to the contralateral (swing leg) knee joint through torque/angular-displacement transfer mechanism during part of its (stance hip) passive extension phase to produce flexion movement at the knee joint of the swing leg.

ii. The torque/angular-displacement transfer mechanism is adjusted to the appropriate 'transfer ratio' so that the desired knee flexion peak attained at an appropriate instant relative to the contralateral (stance leg) hip extension phase.

iii. As in the case of spring brake orthosis (SBO) $[31,32]$, this knee flexion at the swing leg would cause a flexion movement at the ipsilateral hip joint due to existing dynamic coupling between them through a shift in the centre of mass (CoM) of the overall leg segment.

iv. The process raises mechanical energy level of the overall swing leg segment, which is then released as kinetic energy on the occurrence of the desired knee flexion peak by disengaging its coupling with the stance (extending) hip allowing it to swing forward under the influence of the gravity 
and produce an extension movement at the knee joint.

v. An additional knee extension moment is then required to drive the swing knee into full extension and is brought about by reducing the treadmill belt speed, which results in a mechanical energy transfer from the thigh to the shank and progress the shank forward (see below).

\subsubsection{Full knee extension through treadmill speed switch}

When the treadmill belt speed is switched to a lower value, the stance foot resting on it would also go through this sudden decrease of speed. Since the position of the trunk relative to the stance leg would be maintained by the arm-support, the continuity in the hip extension is maintained despite the treadmill belt speed reduction. In practice, this would be brought about at the expense of the arm-support or effort from the subject's upper body. This means that nearly the same velocity reduction is maintained at the proximal end of the swing thigh, while the distal end is still under the influence of the previous higher velocity. This sudden velocity difference at the two ends of the swing thigh segment would give rise to a moment in the direction opposing hip flexion. Now, if the swing leg is assumed to act as a double pendulum configuration (Fig. 2), with the thigh as the top pendulum and shank-foot segment as the bottom pendulum, this hip extension moment would result in a decreasing angular velocity of the top pendulum. As in a double pendulum a decrease in the angular velocity of the top pendulum is always associated with an increase in that of the lower pendulum; $\theta_{2}$ tends to increase (see Fig. 2). Thus, the overall outcome of this reduced treadmill speed would be an additional thrust to the knee extension which would eventually drive the swing knee into full extension.

\subsubsection{Heel strike under the influence of gravity}

Following full knee extension obtained through treadmill speed control and catching it with the knee brake, and perhaps an additional hip flexion following the application of the knee brake, the whole leg segment starts to descent naturally under the influence of gravity and eventually a natural heel strike takes place.

\subsection{Simulation}

A schematic of the simulation environment used in this study is presented in Fig. 3. Various components making up the whole simulation is discussed below with the component being discussed highlighted in italic.

\subsubsection{Various simulation building blocks}

A link segment based full body humanoid forward dynamic model of an average-sized person $(75 \mathrm{~kg}$ mass and $177 \mathrm{~cm}$ height) was developed within vN4D software. In addition to simulating the rigid body dynamics, the software also facilitates the visualization of motion. The model was simplified by limiting the DoF by restraining it to the sagittal plane. Further simplification includes considering the head, arm and trunk as one rigid segment, HAT, resulting in a total of 7 rigid segments, including 3 rigid segments (i.e. thigh, shank and foot) for each leg. These 7 rigid segments interact through 6 hinge joints in the sagittal plane and form the desired link segment model of the full body humanoid with 8 DoF. Anthropometric data for the segments, viz. volume, mass, CoM, joint centre and moment of inertia were obtained from statistical tables based on person's height and weight [33]. The passive viscoelastic properties model for the ankle, knee and hip were taken from the work by Amankwah et al. [34]

Various components of the orthotic support was also implemented within the vN4D software environment. The hip and knee joints could be locked/unlocked feeding control signals from simulink, while the ankle joints were equipped with constant torque springs $(2.5 \mathrm{Nm})$ to hold the feet constantly in a slightly dorsiflexed position, especially during the swing phase flight. To cater for this extra weight that would be incurred by this orthotic arrangement as well as the mounting of the flexible torque transfer mechanism, an extra weight of $5.5 \mathrm{~kg}$ was uniformly added to the leg segments [35]. The locking/unlocking mechanism at the knee and hip joints and the flexible torque transfer mechanism coupling contralateral joints, all implemented within vN4D, could be controlled from the Simulink environment.

The main purpose of the body-weight support (BWS) in the current work was to reduce the magnitude of the whole body mechanical energy fluctuation and hence the required mechanical energy input into the mechanical combination of the subject's body during the whole gait cycle, rather than the usual benefit in varying 'lower extremity loading', which is rendered redundant due to the presence of the orthotic support. Besides, the other benefits of the BWS, e.g. providing assistance to balance by stabilization of the trunk [14], 

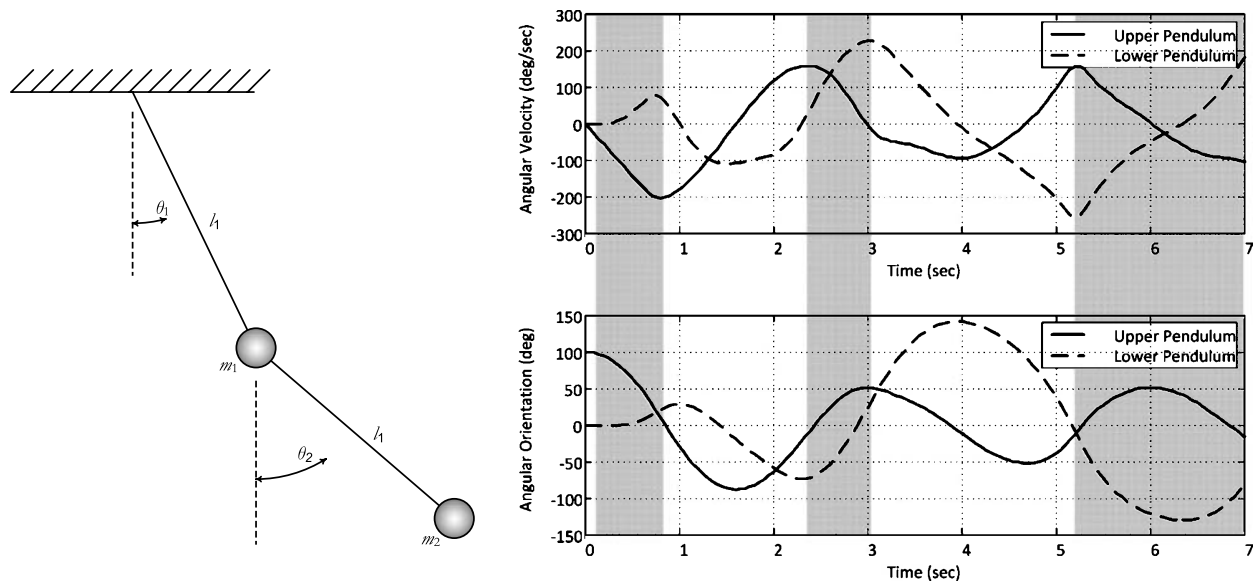

Fig. 2. Angular velocity (upper graph) and angular orientation (lower graph) of the pendula of a typical double pendulum highlighting the periods (shaded area) when the upper pendulum angular velocity is decreasing and is associated with increased angular velocity in the lower pendulum.

Kinematic feedback: $\phi_{\mathrm{H}}, \phi_{\mathrm{K}}, \phi_{\mathrm{A}}$, 'trunk-tilt' etc.

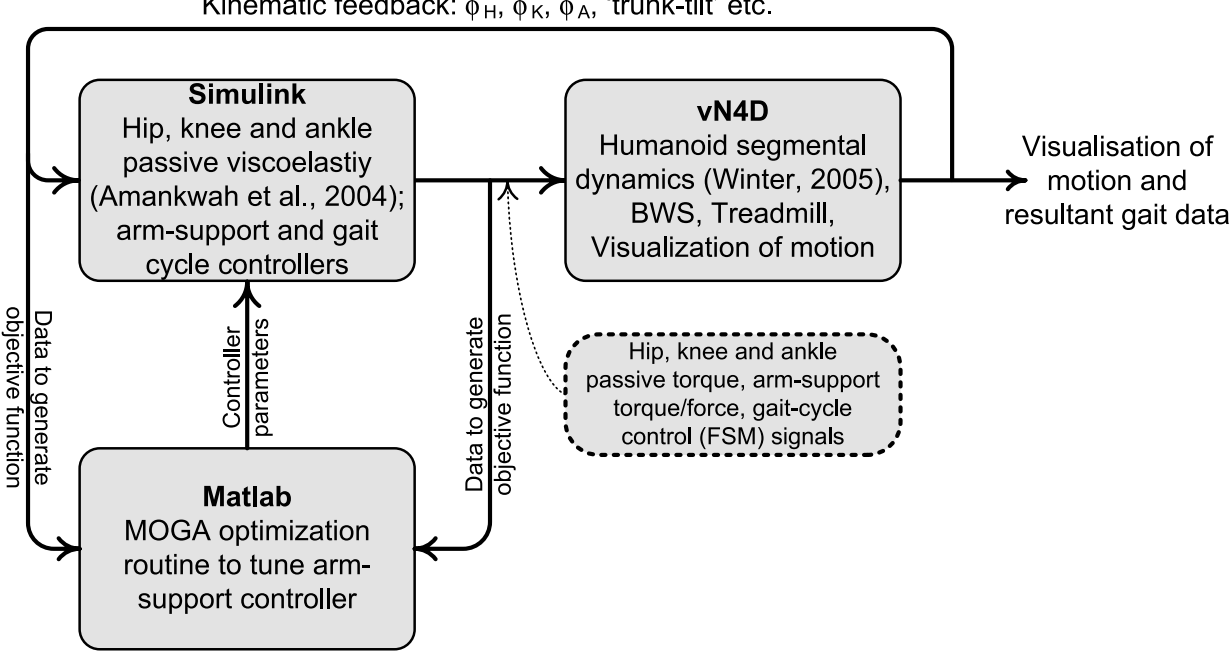

Fig. 3. Simulation environment schematic. $\varphi_{H}, \varphi_{K}$ and $\varphi_{A}$ are hip, knee and ankle angles respectively.

increased safety for the subject [15] would still apply. Following the work of Finch et al. [36], where 30\% BWS condition was found to produce the gait parameters most closely resembling those measured to $0 \%$ (full weight-bearing), 30\% BWS support was implemented within vN4D software environment using two constant force springs connected to an overhead frictionless rolling trolley mechanism [37], each providing an upward pulling force of $110 \mathrm{~N}$, resulting in $30 \%$ BWS for the $75 \mathrm{~kg}$ humanoid.

The treadmill belt was simulated within vN4D software environment using the built-in object- 'conveyer' whose speed could easily be set or controlled from Mat-
lab/Simulink. In light of the analysis on the required friction coefficient $\mu_{r}$ for the proposed fully passive technique of BWSTT [30], the value of the available friction coefficient at the humanoid foot and treadmill belt was set to 0.6. Also to replicate the interaction between the foot and the treadmill belt, the coefficient of restitution (CoR) between them was manually adjusted to a low value $(\sim 0.05)$.

The object-'belt and pulley' within vN4D was used to implement the torque/angular-displacement transfer mechanism, which could easily be activated/deactivated from Simulink. The swing knee is coupled to the contralateral (stance) hip joint during 
part of its extension phase during which the swing knee continues to flex. Thus, the knee flexion peak occurs at the end of that coupled period, while the driving contralateral hip extends by an amount of $\Delta \varphi_{\mathrm{H}, \text { couple }}$. For a given coupling period, and hence $\Delta \varphi_{\mathrm{H} \text {,couple }}$, the magnitude of the knee flexion peak for the swing knee is determined by the transfer ratio $\wp$. With a stance hip flexion angle of $15^{\circ}$ at the opposite toe off and a desired magnitude of knee flexion peak of $60^{\circ}$ for the swing knee, coinciding with a contralateral hip angle of $0^{\circ}\left(\Delta \varphi_{\mathrm{H} \text {, couple }}=15^{\circ}\right)$, the value of the transfer ratio $\wp$ can be worked out as 4 .

Following a trial and error approach, it was found that switching the treadmill speed from $0.9 \mathrm{~m} / \mathrm{s}$ to $0.2 \mathrm{~m} / \mathrm{s}$ at the right instant $\left(\Delta \varphi_{\mathrm{H}, \text { stance }}=-3.5^{\circ}\right)$ provides just enough terminal knee extension drive for the developed model.

\subsubsection{Simulation of arm-support}

To simulate the arm-support for this particular case, first the objectives of the arm-support, as the subject would apprehend them or be instructed to focus their effort to achieve them, are explicitly resolved:

- First and foremost, the arm-support should strive to keep the trunk upright at all times.

- The arm-support should also assist the hip extension through the foot- treadmill belt interaction by resisting any backward movement of the trunk or even push it a bit forward depending on the current angle of the extending stance hip. As a whole, this component of the arm-support would work towards taking the stance hip to desired maximum extension.

The arm-support, which in reality would be delivered from the $2 \times$ elbow and $2 \times$ shoulder joints, are simulated with two PD-type fuzzy logic controllers (FLCs) serving the two objectives as resolved above. The torque/force delivered by these controllers should in effect be equivalent to the effort exerted by the four joints.

A PD-type FLC was deployed to meet the first objective would deliver a torque at the shoulder joint level to keep the trunk upright, the second PD-type FLC delivers force on the trunk along the sagittal plane (in the direction normal to the frontal plane) to achieve the other objective of the arm-support: assisting the passive hip extension mechanism. Accordingly, the actual output for the torque controller is the tilt of the trunk and that of the force controller is the orientation of the extending (stance) hip. Since the stance phase switches between contralateral limbs from step to step during the gait cycle, the FSM discussed later in 2.2.3 is used to realize the switching of the actual output for force controller between contralateral hip joints.

The tuning of the torque controller was achieved through optimization 73 parameters associated with the controller using multi-objective genetic algorithms (MOGA) [38]. The optimality in the solution space was attained by minimizing the trunk tilt (error). Accordingly the first cost or objective function $\mathrm{f}_{1}(y)$ to be

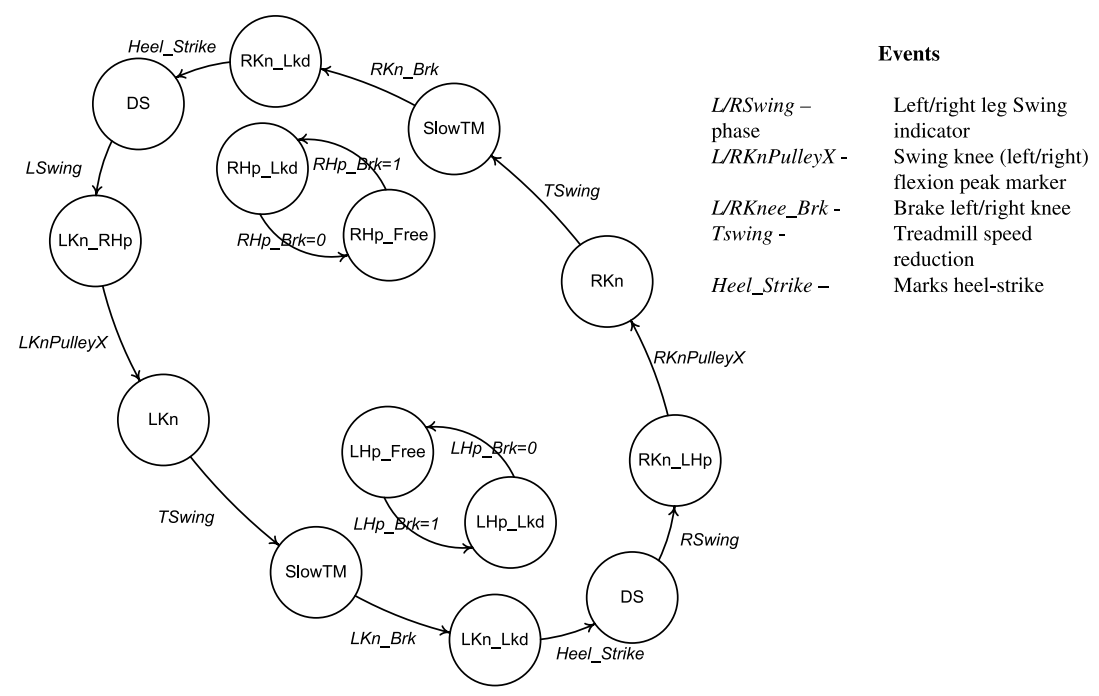

Fig. 4. State graph for the proposed passive treadmill gait cycle controller. 
minimized was defined as the integral square error (ISE):

$$
\mathrm{f}_{1}(y)=\int_{0}^{T}\left(y_{\text {ref }}-y_{\text {act }}\right)^{2} \mathrm{~d} t
$$

where $y_{\text {ref }}$ and $y_{\text {act }}$ are the reference input $\left(0^{\circ}\right.$ for the upright trunk) and the actual output (trunk tilt) respectively.

To mimic the prominent feature associated with any natural human activity: minimizing the energy cost [39], the time integral of the absolute value of the torque is minimized as much as possible without penalizing its actual purpose (keeping the trunk upright). Hence a second objective function $\mathrm{f}_{2}(y)$ to be minimized was defined as the integral square control toque (ISCT) delivered by the controller over a complete gait cycle ( $\mathrm{T} \mathrm{sec})$ :

$$
\mathrm{f}_{2}(y)=\int_{0}^{T} u^{2} \mathrm{~d} t
$$

where $u$ is the control variable or the torque delivered by the controller. A MOGA routine, written in Matlab, with a population size of 50 binary coded individuals in each generation was run for 45 generations to tune the parameters of the FLC through minimizing both the objective functions independently and simultaneously. The genetic operators of the MOGA, the crossover and mutation rate were initialized as $80 \%$ and 0.01 respectively. The ranges of search or the 'feasible space' for the parameters were set by using a trial and error approach. The force FLC, with a reference input of maximum hip extension angle, on the other hand, was tuned heuristically.

\subsubsection{Synthesis of gait cycle control}

The control of the proposed gait cycle basically comprises coordinated activation and deactivation of the braking mechanisms mounted at the hip and the knee joints as well as the torque/angular-displacement transfer mechanisms coupling both knee joints to their contralateral hip joints. A finite state controller or machine (FSM) [40] was designed for this purpose. The events determining the transition between states were detected through combination of kinematic data fed back from the humanoid. The whole gait cycle is decomposed into a sequence of ten states with four additional parallel states (Fig. 4). The sequence of states involved in the implementation of the proposed gait cycle is shown schematically in Fig. 4 for $50 \%$ of the gait cycle along with the list of encoded events. As a first step towards the synthesis of the FSM, the events causing these state transitions (for $50 \%$ of the gait cycle) are encoded as follows:

\section{Right leg Swing phase indicator RSwing}

This event is set following the double-support (DS) state has been attended for 0.2 seconds with right foot behind the left foot. RSwing causes the FSM to enter the state 'RKn_LHp', which ultimately releases the brake at the right knee joint and activates its coupling with the contralateral hip joint and thus initiates the swing of the right leg. The rising edge of the signal also resets the treadmill speed to its normal (higher) value. The signal is held set until the end of right swing phase, when it is reset by the Heel_Strike event.

\section{Swing knee flexion peak marker- $R K n$ Pulley $X$}

This event basically signals the FSM to decouple the right knee from the contralateral (stance) hip joint during its swing phase at an instant when the knee flexion amounts to the desired knee flexion peak. As discussed in 2.1, the desired knee flexion peak occurs when the contralateral hip joint attains an angle of $0^{\circ}$ during its extension phase and hence the event is detected as:

$$
R \text { Swing AND (Hip-Angle < 0) }
$$

The FSM thus enters the 'RKn' state at the rising edge of the signal $R K n$ Pulley $X$ through releasing the coupling between the contralateral joints.

\section{Braking right knee with $\mathrm{RKnee}_{-} \mathrm{Brk}$}

This event/input to the FSM is generated when the swing knee reaches its full or maximum extension as follows:

(Knee-angle $\leq 2)$ AND (Knee-angular-velocity $\geq$ $0^{\circ} / \mathrm{sec}$ ) AND (Hip-angle $\geq 15^{\circ}$ )

\section{Treadmill speed reduction with TSwing}

The FSM slows down the treadmill to provide the swing knee with terminal extension drive on occurrence of this event. As described in 2.2, the event is detected at an appropriate instant as:

$$
R \text { Swing AND (Left-hip-angle }>-3.5^{\circ} \text { ) }
$$

The treadmill speed is accordingly slowed down on occurrence of TSwing and the FSM makes transition to 'SlowTM' state. 

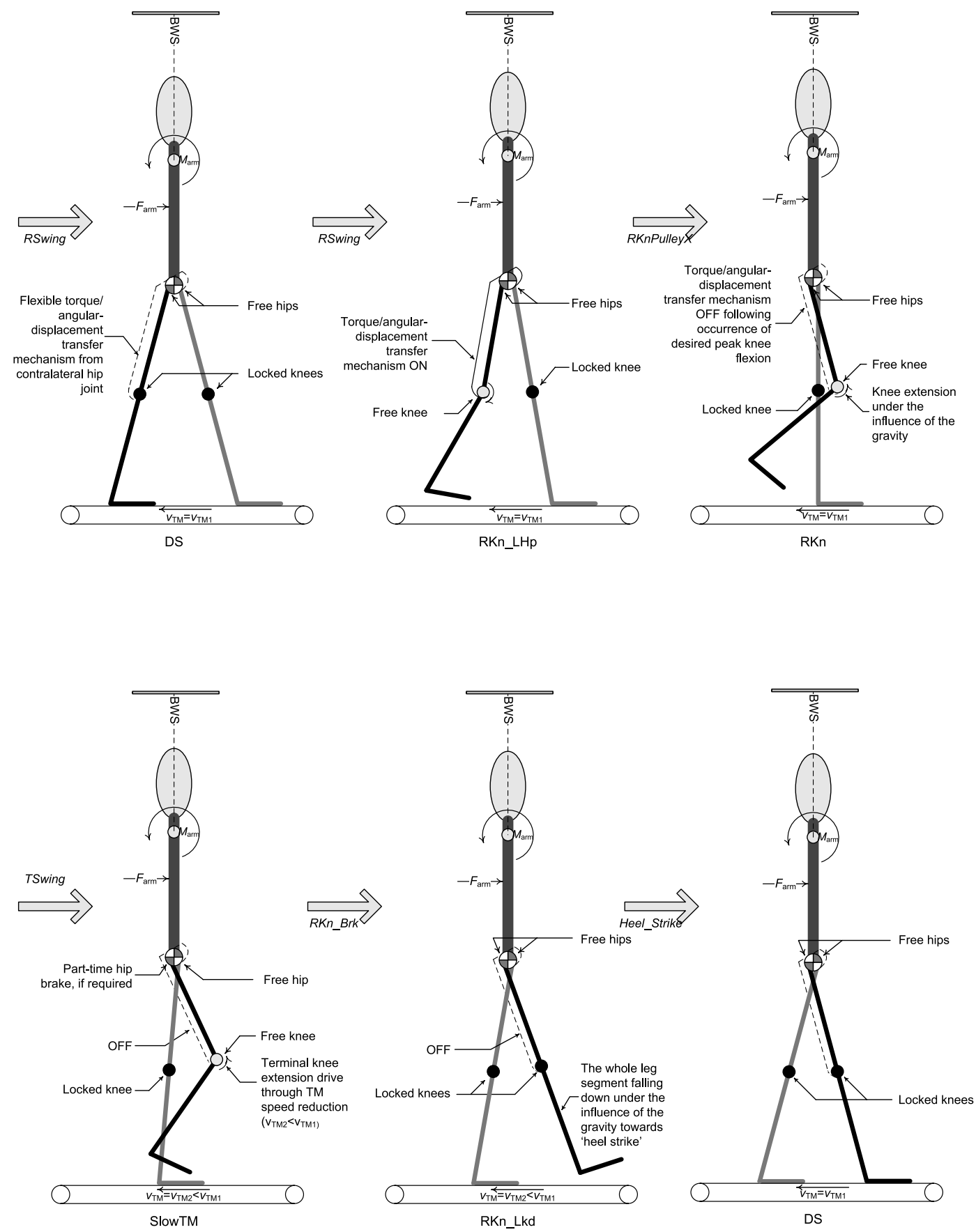

Fig. 5. Sequence of states during $50 \%$ of the gait cycle.

\section{Heel_Strike}

The event arises when the foot touches the ground at the end of swing phase and is detected within the simulation environment simply by observing the distance between the treadmill belt and the left foot following hip flexion peak in the swing leg. While for the right leg, the event is detected as follows, and the final signal is generated as an OR combination of the event from both the legs. 
Table 1

Summary of states and transition rules during $50 \%$ of the gait cycle

\begin{tabular}{|c|c|c|}
\hline State & Rules & \\
\hline \multirow[t]{2}{*}{ DS (Double-support) } & Entry & $\begin{array}{l}\text { - Reset } \mathbf{L S w i n g} / \mathbf{R S w i n g} \\
\text { - Restore treadmill velocity to } v_{T M 1} \\
\text { - Wait } 0.2 \text { seconds }\end{array}$ \\
\hline & Transit & • IF RSwing THEN transit RKn_LHp \\
\hline \multirow[t]{2}{*}{ RKn_LHp (Right knee coupled to left hip) } & Entry & $\begin{array}{l}\text { - Release right knee brake (reset } \mathbf{R K n} \_ \text {Brk) } \\
\text { - Activate contralateral torque transmission }\end{array}$ \\
\hline & Transit & • IF $\boldsymbol{R} \boldsymbol{K} \boldsymbol{n}$ Pulley $\boldsymbol{X}$ THEN transit RKn \\
\hline RKn (Right knee free, decoupled from left hip) & $\begin{array}{l}\text { Entry } \\
\text { Transit }\end{array}$ & $\begin{array}{l}\text { - Release right knee or contralateral torque transmission } \\
\text { - IF } \boldsymbol{T S}_{\text {wing }} \text { THEN transit SlowTM }\end{array}$ \\
\hline SlowTM (Reduced treadmill speed) & $\begin{array}{l}\text { Entry } \\
\text { Transit }\end{array}$ & $\begin{array}{l}\text { - Reduce treadmill velocity } v_{T M 1} \text { to } v_{T M 2} \\
\text { - IF } \boldsymbol{R} \boldsymbol{K} \boldsymbol{n}_{-} \boldsymbol{B} \boldsymbol{r} \boldsymbol{k} \text { THEN transit RKn_Lkd }\end{array}$ \\
\hline \multirow[t]{2}{*}{ RKn_Lkd (Right knee fully extended and locked) } & Entry & $\begin{array}{l}\text { - Reset LSwing/Rswing } \\
\text { - Activate right knee brake }\end{array}$ \\
\hline & Transit & - IF $\boldsymbol{R}$ Swing THEN transit RKn_LHp \\
\hline \multirow[t]{2}{*}{ RHp Free (Right hip free) } & Entry & $\begin{array}{l}\text { - Reset } \mathbf{L S w i n g} / \mathbf{R s w i n g} \\
\text { - Restore treadmill velocity to } v_{T M 1} \\
\text { - Wait } 0.2 \text { seconds }\end{array}$ \\
\hline & Transit & - IF RSwing THEN transit RKn_LHp \\
\hline \multirow[t]{2}{*}{ RHp_Lkd (Right hip locked) } & Entry & - Apply right hip brake \\
\hline & Transit & - IF $\boldsymbol{R} \boldsymbol{H} \boldsymbol{p}_{-} \boldsymbol{B} \boldsymbol{r k}=0$ THEN transit $\mathbf{R H p}$ _Free \\
\hline
\end{tabular}

(treadmill-belt $\rightarrow$ Right-foot vertical distance $\geq 0.05$ meter) AND (Right-hip-angle $\geq 15^{\circ}$ )

\section{Parallel state: Braking right hip with $\mathrm{RH}_{\mathrm{H}} \mathrm{Brk}$}

Within the $50 \%$ gait cycle discussed above, there is another event $\mathrm{RH}_{\mathrm{H}} \mathrm{Brk}$, which is associated with the occasional transition to a parallel state 'RHp_Lkd'. The event detector generates this input to brake the swing hip, if required, to maintain its flexion during the swing knee extension phase to ensure adequate foot-ground clearance. The algorithm behind the event is to lock the hip joint in the swing leg following the passive hip flexion peak (during the knee extension phase) if the knee still has more than $25^{\circ}$ to reach its full extension, while the hip flexion angle is $30^{\circ}$ or less with negative angular velocity (extending):

(Right-hip-angle $\leq 30^{\circ}$ ) AND (Right-hip-velocity $\geq-5^{\circ} / \mathrm{sec}$ ) AND (Right-knee-angle $\geq 25^{\circ}$ )

A summary of the states and associated rules for $50 \%$ of the gait cycle is presented in Table 1. A pictorial representation of the states and transitions, based on approximate joint kinematics, is presented in Fig. 5 for $50 \%$ of the gait cycle.

\section{Results}

Following the initial tuning of the arm-support controllers, the FSM was able to simulate unlimited continuous near natural treadmill gait. Considering the two primary aspects of the simulation study, viz. the simulation of the arm-support and the implementation of the overall gait cycle with FSM, the results are organized into two subsections and are presented below.

\subsection{Arm support simulation}

Fig. 6 shows the non-dominated solution fronts after certain generations for the multi-objective optimization of the torque controller with the ISE as one of the objectives $\mathrm{f}_{1}(y)$ [Equation 1] and ISCT as the second objective $\mathrm{f}_{2}(y)$ [Equation 2]. The figure contains two solution fronts obtained after 20 and 45 generations. With the solution front after 20 generations fully dominated by the front after 45 generations, the result clearly manifests convergence of the solutions with generations. Since $\mathrm{f}_{1}(y)$ basically caters for the main purpose of the arm-support, the solution set (controller parameters) corresponding to the best $\mathrm{f}_{1}(y)$ (indicated with an arrow in Fig. 6) was chosen for the simulation of the arm-support.

Fig. 7 (a) shows the output from the torque controller or the simulated arm-support component responsible 


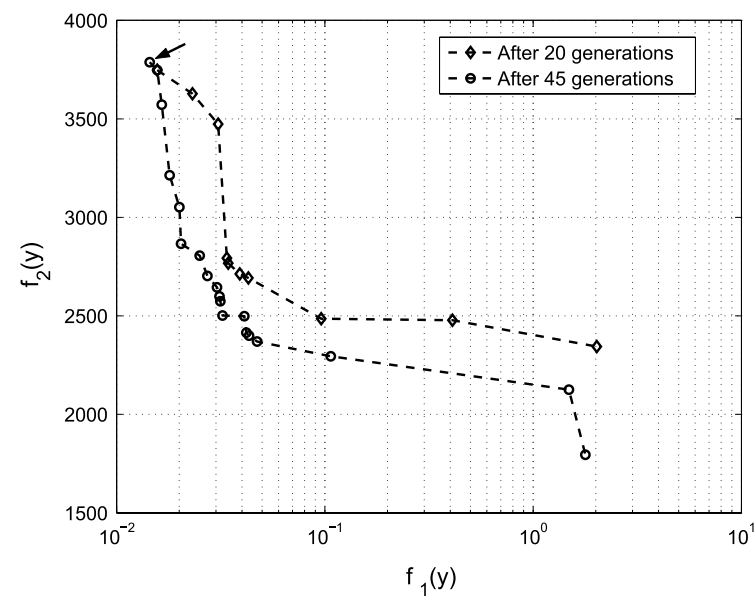

Fig. 6. Non-dominated solution fronts for shoulder joint controller tuning with MOGA after 20 and 45 generations. The cost-functions $\mathrm{f}_{1}(y)$ and $f_{2}(y)$ are defined in Equation (1) and Equation (2) respectively.
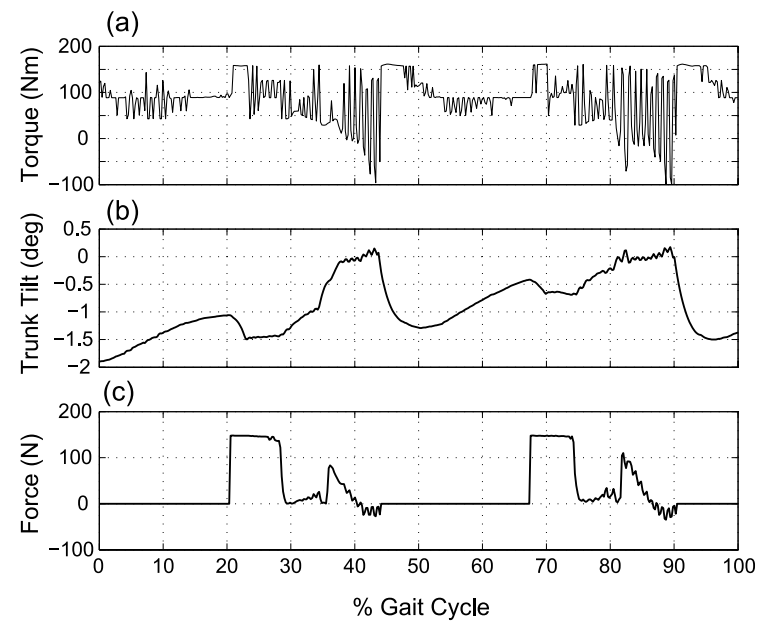

Fig. 7. Arm-support results: (a) output from the torque controller, (b) Trunk tilt or actual output, and (c) output from force controller.

for maintaining the trunk upright along with the tilt of the trunk (actual output) for a complete gait cycle, Fig. 7 (b). According to the sign convention followed in this work, a positive tilt of the trunk indicates a backwards inclination. Thus a positive shoulder joint torque would produce a positive trunk tilt or resist a negative tilt (forward inclination of the trunk).

The other component of the arm-support whose main purpose is to apply force on the trunk so as to assist the hip extension process of the stance hip is shown Fig. 7 (c). Ranging from $-34.6 \mathrm{~N}$ to $148 \mathrm{~N}$, the predominantly positive force has a mean value of 49.34 $\mathrm{N}$, meaning that this component of the arm-support acts mostly to push the body forward.

\subsection{Gait cycle generation with finite state machine}

Following successful tuning of the arm-support controllers, the whole model was able to run indefinitely, simulating arbitrarily large number of continuous gait cycles without any interruption. The simulation was able to run for over 500 continuous gait cycles following which it was stopped manually. Due to the computationally intensive nature of the simulation environment, consisting of Matlab in conjunction with vN4D, it took almost 2 minutes to simulate just a single gait cycle ( $3.1 \mathrm{sec}$ of real time) on a $1.4 \mathrm{GHz}$ Pentium4 processor with 1GB RAM. For analysis purposes, 
Table 2

Spatio-temporal parameters of the resultant gait

\begin{tabular}{lll}
\hline Parameter & Value (mean) & Variance \\
\hline Velocity $(\mathrm{m} / \mathrm{s})$ & 0.4401 & N/A \\
Cadence (steps/min) & 38.6 & N/A \\
Opposite Toe Off $(\%$ gait cycle) & 12.826 & 0.004 \\
Duration of Stance $(\%$ gait cycle) & 63.028 & 0.0043 \\
Swing (\% gait cycle) & 36.656 & 0.0038 \\
Stride Length $(\mathrm{cm})$ & 136.87 & 0.0042 \\
\hline
\end{tabular}

we concentrate on a single gait cycle in this section. All the results have been presented with their time axis normalized to one gait cycle.

The resultant lower limb joint kinematics along with the event detector outputs from the finite state machine are shown in Fig. 8 for a single gait cycle. The left column in the figure results from one leg while the right column results from the opposite leg. The stick diagram on the top row of the figure indicates the approximate kinematic orientation of the subject at the corresponding instant of the gait cycle.

With a view to highlight (with shaded area) the coupling phenomenon of the contralateral hip-knee joints, hip and knee joint trajectories, along with those of the opposites are plotted in Fig. 9. The area under each shaded area is defined by the mutually coupled trajectories and the duration of the coupling. The figure also depicts the associated outputs from the event detector - on/off switching the coupling.

The ground clearance resulting from the swinging leg is presented in Fig. 10 where it was measured as the closest foot-treadmill distance attained by each foot during the course of the gait cycle within the vN4D environment. With an average clearance of $3 \mathrm{~cm}$ during the mid-swing, it is evident that the proposed method is able to produce adequate ground clearance for the progression of the swing leg.

The spatiotemporal gait parameters tabulated in Table 2 is obtained observing ten consecutive gait cycles. The observed variance had a very low value, reasserting the repeatability of the overall method.

\section{Discussion}

Even though the torque from the arm-support agents fluctuates between $-99.53 \mathrm{Nm}$ to $161.4 \mathrm{Nm}$ within the gait cycle (Fig. 7-a), it may be noticed that the torque is mostly positive with a significant positive mean value of $82.36 \mathrm{Nm}$ over the gait cycle. This, according to the sign convention, means that during the resultant gait cycle, the upper-body would normally tend to lean forward and thus the major role of this particular component of the arm-support would mainly be to prevent the upper-body from leaning forward. This predominantly positive nature of the torque component also indicates the possibility of partially assisting the arm-support with passive elastic mechanism carefully positioned/mounted on the treadmill facility, thus reducing the burden on the arm-support.

Force/torque magnitude aside, the metabolic cost incurred by the arm-support is another crucial factor determining the practicality of the proposed technique. Within the simulation environment, it was not possible to account for the metabolic cost incurred by the armsupport and can only be determined through practical setup (e.g. metabolic gas measure) [41].

Although the magnitude of the optimized armsupport (output of the FLCs) seems to be within the capability of the combined four arm joints of a paraplegic subject with functional upper body, the real scenario can be expected to be better, as the human intelligence would in reality be replacing the MOGA optimization carried out in this work.

The gait cycle generation results confirm that, as expected according to the design objective (2.2), the peak knee flexion during the swing phase was $\sim 65^{\circ}$ (Figs. 8 and 9). But unlike the SBO generated swing phase, the rising part of the swing knee joint trajectory was found to be almost linear with time. This linearity in the trajectory results from its coupling with the contralateral hip joint and its extension trajectory. Fig. 9, where the segments of trajectories during which the coupling is on are highlighted (shaded area), depicts this phenomenon more clearly.

It may be noted in Fig. 8 that the signals to brake the hip, $L H p_{-} B r k / R H p_{-} B r k$ are zero at all times, indicating a free hip joint throughout the gait cycle. Yet, with a peak hip flexion of around $30^{\circ}$, the resultant hip joint trajectory is quite within acceptable (natural) range; especially during the swing phase [33]. Although the passive flexion mechanism of the swing hip has been accounted for as a consequence of its dynamic coupling with the ipsilateral flexing knee joint, the dynamic simulation shows that the hip joint continues to flex even after the ipsilateral knee starts to extend following the flexion peak. This is also evident from the kinematics in Fig. 8. The treadmill speed reduction with the offset of the TSwing event to provide the swing knee with its terminal drive was found to serve an additional purpose of preventing excessive hip flexion. The coincidence of 


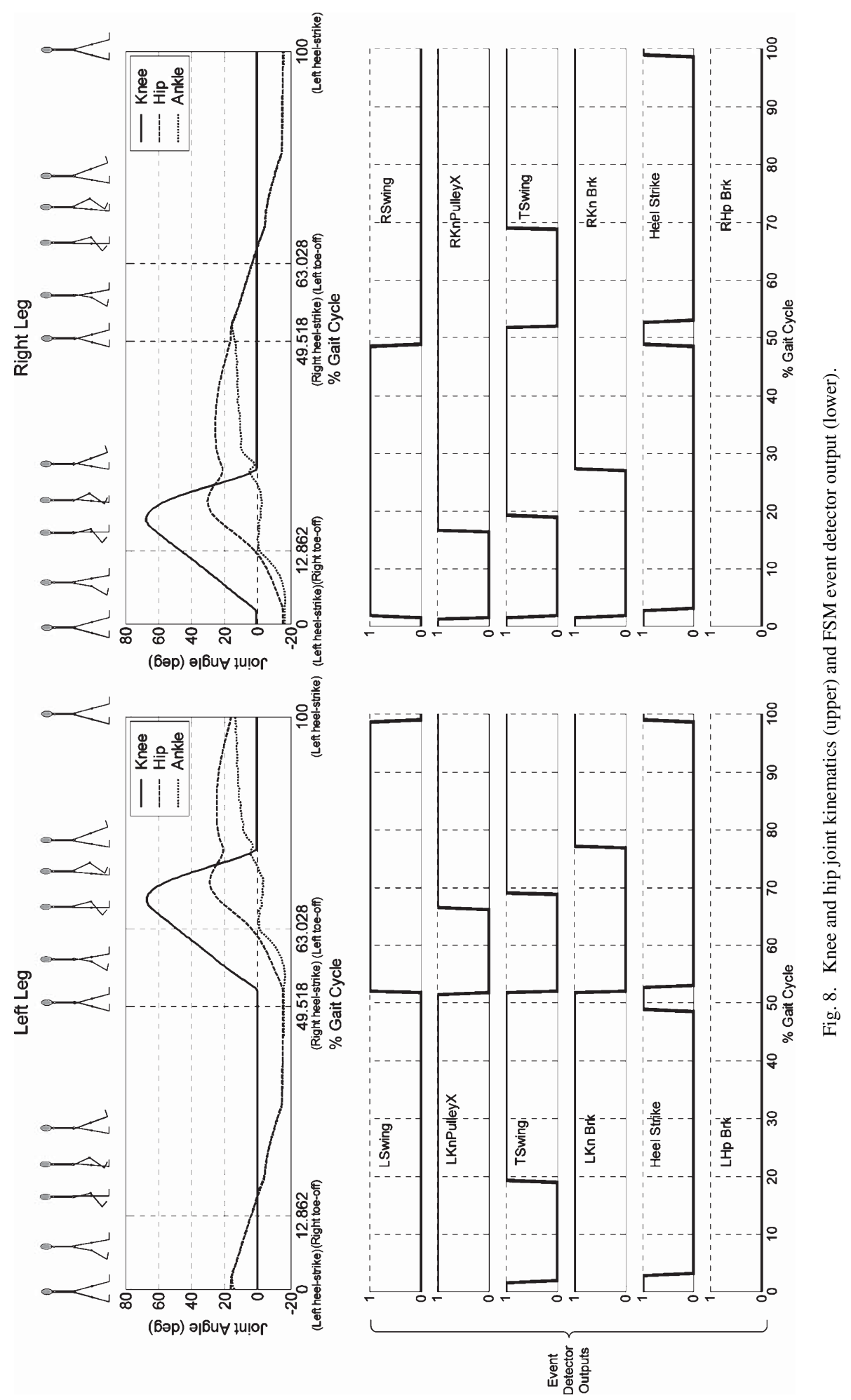



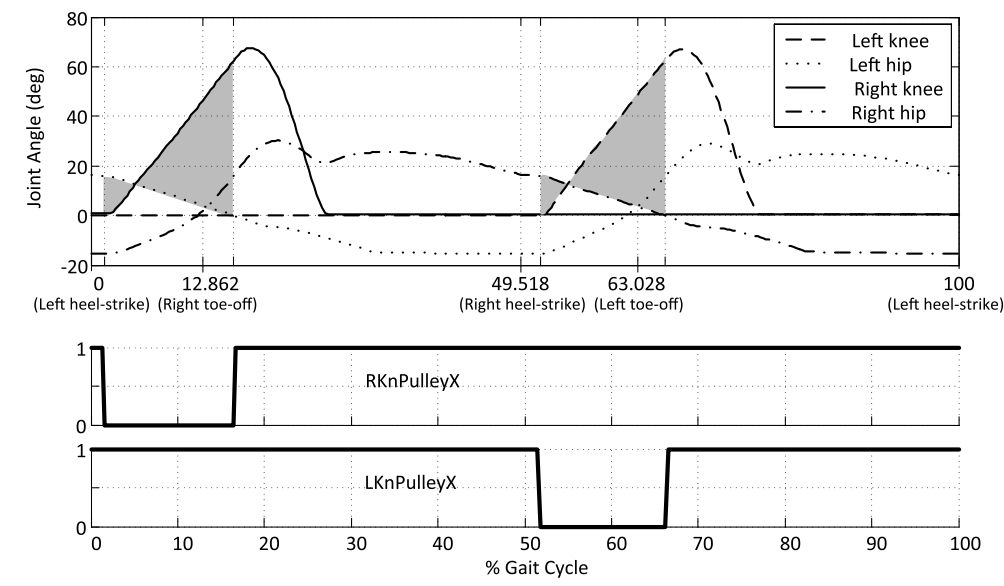

Fig. 9. Joint kinematics highlighting (shaded area) the coupling of the knee joint with the contralateral hip joint (above) and associated signals from the event detector.

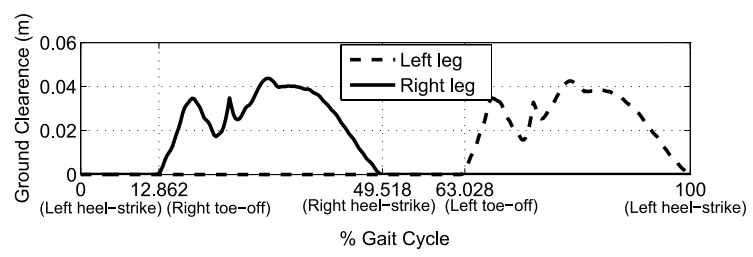

Fig. 10. Ground clearance during gait cycle.

the hip flexion peak with the offset of the TSwing signal is an indicative of this phenomenon. The swing hip trajectory is found to be affected by the occurrence of the full knee extension, as the extending shank, because of its momentum tends to push the thigh forward once the full knee extension is reached and the knee joint is locked. This results in a secondary bump in the swing hip trajectory following its flexion peak.

The proposed idea of attaching the ankle joint with constant torque spring to provide slight dorsiflexion at all times seems to work perfectly with the generated gait cycle through $(i)$ catering for adequate ankle joint freedom during the stance phase, as well as (ii) ensuring ground clearance by providing dorsiflexion during the swing flight. The ankle joint kinematics in Fig. 8 show dorsiflexion during the initial swing flight, until suddenly the ankle joint starts losing its dorsiflexion and attains slightly planterflexed orientation. This is caused by the sudden treadmill speed reduction and hence can be seen to coincide with the switching of the TSwing signal. The treadmill speed reduction has already been pointed out as an agent limiting the hip flexion of the swing leg. This, being combined with the planter flexing ankle would obviously affect the ground clearance adversely. The effect can be observed in Fig. 10 where the ground clearance measured as the closest foot-treadmill distance, attained by each foot is shown for one complete gait cycle. From this plot, it is prominent that the ground clearance is suddenly forced drastically low during its pick up phase as a result of the treadmill speed reduction. Despite this adverse effect, the resultant ground clearance appears to be quite acceptable for a sound gait cycle.

The only purpose of the hip brake was to ensure adequate ground clearance in certain hypothetical kinematic conditions. The simulation suggests no possibility of that situation indicating the provision of the hip brake to be potentially redundant. This can only be confirmed through further investigation, perhaps through practical implementation, and could pave the way to further simplification of the method both in terms of physical orthosis and control.

It was beyond the scope of this work to estimate the potential metabolic energy cost incurred on the subject, which may turn out to be a key factor in implementing the technique in practice. In the adverse event of intolerable (too high) level of metabolic cost, the results also indicate possible solution through orthot- 
ically supporting the trunk to prevent forward tilt. Moreover, it might be possible to substitute the knee extension through treadmill speed reduction by FES of the quadriceps $[31,32]$, in which case the technique would not remain entirely passive anymore. In either case there are advantages and disadvantages associated with various aspects, and thus would be a matter of choice depending on the available resources.

\section{Conclusions}

The overall objective of the simulation study was to cater for an inexpensive yet flexible test environment which would enable a successful initial study of the proposed idea. While, in general, simulation platform such as this has its own merits, e.g. the engineers to gain some crucial prior insight into certain features of the actual plant and thereby make some informed decisions prior to the actual implementation, the actual engineering work of developing the real plant has its own challenges per se. Certainly such methods require intensive engineering work to be implemented in practice, and hence play a key role in the overall success of the method. Nonetheless, it may safely be affirmed that this simulation study has revealed some optimistic results relating to the proposed BWSTT, which renders it potentially worthwhile to be implemented in reality and eventually its actual performance being evaluated.

\section{References}

[1] B.H. Dobkin, S. Harkema, P. Requejo, V.R. Edgerton, et al. Modulation of locomotor-like emg activity in subjects with complete and incomplete spinal cord injury, Journal of Neurologic Rehabilitation 9(4) (1995), 183.

[2] H. Barbeau and S. Rossignol, Recovery of locomotion after chronic spinalization in the adult cat, Brain Res 412(1) (1987), 84-95. 0006-8993 (Print) Journal Article Research Support, Non-U.S. Gov't.

[3] H. Barbeau, M. Landouceur, K.E. Norman, A. Pépin and A. Leroux, Walking after spinal cord injury: Evaluation, treatment, and functional recovery, Archives of Physical Medicine and Rehabilitation 80(2) (1999), 225-235.

[4] A.L. Behrman and S.J. Harkema, Locomotor training after human spinal cord injury: A series of case studies, Physical Therapy 80(7) (2000), 688-700.

[5] E.C. Field-Fote, Spinal cord control of movement: implications for locomotor rehabilitation following spinal cord injury, Physical Therapy 80(5) (2000), 477-484.

[6] I.T. da Cunha Filho, P.A.C. Lim, H. Qureshy, H. Henson, T. Monga and E.J. Protas, A comparison of regular rehabilitation and regular rehabilitation with supported treadmill ambulation training for acute stroke patients, Journal of Rehabilitation Research and Development 38(2) (2001), 245256.

[7] Y. Laufer, R. Dickstein, Y. Chefez and E. Marcovitz, The effect of treadmill training on the ambulation of stroke survivors in the early stages of rehabilitation, J Rehabil Res Dev 38 (2001), 69-78.

[8] I. da Cunha, P.A. Lim, H. Qureshy, H. Henson, T. Monga, E.J. Protas, et al. Gait outcomes after acute stroke rehabilitation with supported treadmill ambulation training: a randomized controlled pilot study, Archives of Physical Medicine and Rehabilitation 83(9) (2002), 1258-1265.

[9] M. Visintin, H. Barbeau, N. Korner-Bitensky and N.E. Mayo, A new approach to retrain gait in stroke patients through body weight support and treadmill stimulation, Stroke 29(6) (1998), 1122-1128.

[10] S. Hesse, C. Bertelt, A. Schaffrin, M. Malezic, K.-H. Mauritz, et al. Restoration of gait in nonambulatory hemiparetic patients by treadmill training with partial body-weight support, Archives of Physical Medicine and Rehabilitation 75(10) (1994), 1087.

[11] I. Cikajlo, Z. Matjacic and T. Bajd, A treadmill based swing phase re-education. In Neural Engineering, 2003. Conference Proceedings. First International IEEE EMBS Conference on, IEEE, 2003, 257-260.

[12] S. Hesse, T.H. Sarkodie-Gyan and D. Uhlenbrock, Development of an advanced mechanised gait trainer, controlling movement of the centre of mass, for restoring gait in nonambulant subjects-weiterentwicklung eines mechanisierten gangtrainers mit steuerung des massenschwerpunktes zur gangrehabilitation roll-stuhlpichtiger patienten, Biomedizinische Technik/Biomedical Engineering 44(7-8) (1999), 194-201.

[13] S. Hesse, C. Bertelt, M.T. Jahnke, A. Schaffrin, P. Baake, M. Malezic and K.H. Mauritz, Treadmill training with partial body weight support compared with physiotherapy in nonambulatory hemiparetic patients, Stroke 26(6) (1995), 976-981.

[14] E.C. Field-Fote, Combined use of body weight support, functional electric stimulation, and treadmill training to improve walking ability in individuals with chronic incomplete spinal cord injury, Archives of Physical Medicine and Rehabilitation 82(6) (2001), 818-824.

[15] N.J. Postans, J.P. Hasler, M.H. Granat and D.J. Maxwell, Functional electric stimulation to augment partial weightbearing supported treadmill training for patients with acute incomplete spinal cord injury: A pilot study, Arch Phys Med Rehabil 85(4) (2004), 604-610.

[16] S. Hesse, H. Schmidt, C. Werner and A. Bardeleben, Upper and lower extremity robotic devices for rehabilitation and for studying motor control, Current Opinion in Neurology 16(6) (2003), 705-710.

[17] S. Hesse, D. Uhlenbrock, et al. A mechanized gait trainer for restoration of gait, Journal of Rehabilitation Research and Development 37(6) (2000), 701-708.

[18] H. Schmidt, S. Hesse, C. Werner and A. Bardeleben, Upper and lower extremity robotic devices to promote motor recovery after stroke-recent developments, In Engineering in Medicine and Biology Society, 2004. IEMBS'04. 26th Annual International Conference of the IEEE, IEEE, 2004, volume 2, pp. 4825-4828.

[19] G. Colombo, M. Joerg, R. Schreier, V. Dietz, et al. Treadmill training of paraplegic patients using a robotic orthosis, 
Journal of rehabilitation research and development 37(6) (2000), 693-700.

[20] G. Colombo, M. Wirz, V. Dietz, et al. Driven gait orthosis for improvement of locomotor training in paraplegic patients, Spinal Cord 39(5) (2001), 252-255.

[21] T. George Hornby, D.H. Zemon and D. Campbell, Robotic-assisted, body-weight-supported treadmill training in individuals following motor incomplete spinal cord injury, Physical Therapy 85(1) (2005), 52-66.

[22] D. Reinkensmeyer, J.H. Wynne and S.J. Harkema, A robotic tool for studying locomotor adaptation and rehabilitation. In Engineering in Medicine and Biology, 2002. 24th Annual Conference and the Annual Fall Meeting of the Biomedical Engineering Society EMBS/BMES Conference, 2002. Proceedings of the Second Joint, IEEE, 2002, volume 3, pp. 2353-2354.

[23] W.E. Ichinose, D.J. Reinkensmeyer, D. Aoyagi, J.T. Lin, K. Ngai, V. Reggie Edgerton, S.J. Harkema and J.E. Bobrow, A robotic device for measuring and controlling pelvic motion during locomotor rehabilitation. In Engineering in Medicine and Biology Society, 2003. Proceedings of the 25th Annual International Conference of the IEEE, IEEE, 2003, volume 2, pp. 1690-1693.

[24] H. Schmidt, D. Sorowka, S. Hesse and R. Bernhardt, Design of a robotic walking simulator for neurological rehabilitation. In Intelligent Robots and Systems, 2002. IEEE/RSJ International Conference on, IEEE, 2002, volume 2, pp. 1487-1492.

[25] C.J. Bosecker and H.I. Krebs, Mit-skywalker. In Rehabilitation Robotics, 2009. ICORR 2009. IEEE International Conference on, IEEE, 2009, pp. 542-549.

[26] S. Collins, A. Ruina, R. Tedrake and M. Wisse, Effcient bipedal robots based on passive-dynamic walkers, Science 307(5712) (2005), 1082-1085.

[27] E.B. MacKnight, M.R. Popovic and T. Adam Thrasher, Functional electrical therapy for assisted treadmill training: use of passive dynamics. In Engineering in Medicine and Biology Society, 2003. Proceedings of the 25th Annual International Conference of the IEEE, IEEE, 2003, volume 2, pp. 15231526.

[28] K.-H. Mauritz, Gait training in hemiplegia, European Journal of Neurology 9(s1) (2002), 23-29.

[29] I. Cikajlo, Z. Matjačić, T. Bajd and R. Futami, Sensory supported FES control in gait training of incomplete spinal cord injury persons, Artificial Organs 29(6) (2005), 459-461.

[30] M.S. Huq, Analysis and Control of Hybrid Orthosis in Therapeutic Treadmill Locomotion for Paraplegia. $\mathrm{PhD}$ thesis, Department of Automatic Control and Systems Engineering, University of Sheffeld, 2009.
[31] M.S. Huq and M.O. Tokhi, Genetic algorithms based approach for designing spring brake orthosis-part I: Spring parameters, Applied Bionics and Biomechanics 9(3) (2012), 303-316.

[32] M.S. Huq and M.O. Tokhi, Genetic algorithms based approach for designing spring brake orthosis -part II: Control of fes induced movement, Applied Bionics and Biomechanics 9(3) (2012), 317-331.

[33] D.A. Winter, Biomechanics and motor control of human movement, Wiley (2009).

[34] K. Amankwah, R.J. Triolo and R. Kirsch, Effects of spinal cord injury on lower-limb passive joint moments revealed through a nonlinear viscoelastic model, Journal of Rehabilitation Research and Development 41(1) (2004), 15-32.

[35] C.S. To, R.F. Kirsch, R. Kobetic and R.J. Triolo, Simulation of a functional neuromus-cular stimulation powered mechanical gait orthosis with coordinated joint locking, Neural Systems and Rehabilitation Engineering, IEEE Transactions on 13(2) (2005), 227-235.

[36] L. Finch, H. Barbeau and B. Arsenault, Inuence of body weight support on normal human gait: development of a gait retraining strategy, Physical Therapy 71(11) (1991), 842855.

[37] M.S. Wilson, H. Qureshy, E.J. Protas, S. Ann Holmes, T.A. Krouskop and A.M. Sherwood, et al. Equipment specifications for supported treadmill ambulation training, Journal of Rehabilitation Research and Development 37(4) (2000), 415-422.

[38] C.M. Fonseca, P.J. Fleming, et al. Genetic algorithms for multiobjective optimization: For-mulation, discussion and generalization. In Proceedings of the fifth international conference on genetic algorithms, San Mateo, California, volume 1, p. 416, 1993.

[39] V.T. Inman, Human locomotion, Canadian Medical Association Journal 94(20) (1966), 1047.

[40] P.C. Sweeney, G.M. Lyons and P.H. Veltink, Finite state control of functional electrical stimulation for the rehabilitation of gait, Medical and Biological Engineering and Computing 38(2) (2000), 121-126.

[41] J. Beillot, F. Carre, G. Le Claire, P. Thoumie, B. PerruoinVerbe, A. Cormerais, A. Courtillon, E. Tanguy, G. Nadeau, P Rochcongar, et al. Energy consumption of paraplegic locomotion using reciprocating gait orthosis, European Journal of Applied Physiology and Occupational Physiology 73(3) (1996), 376-381. 

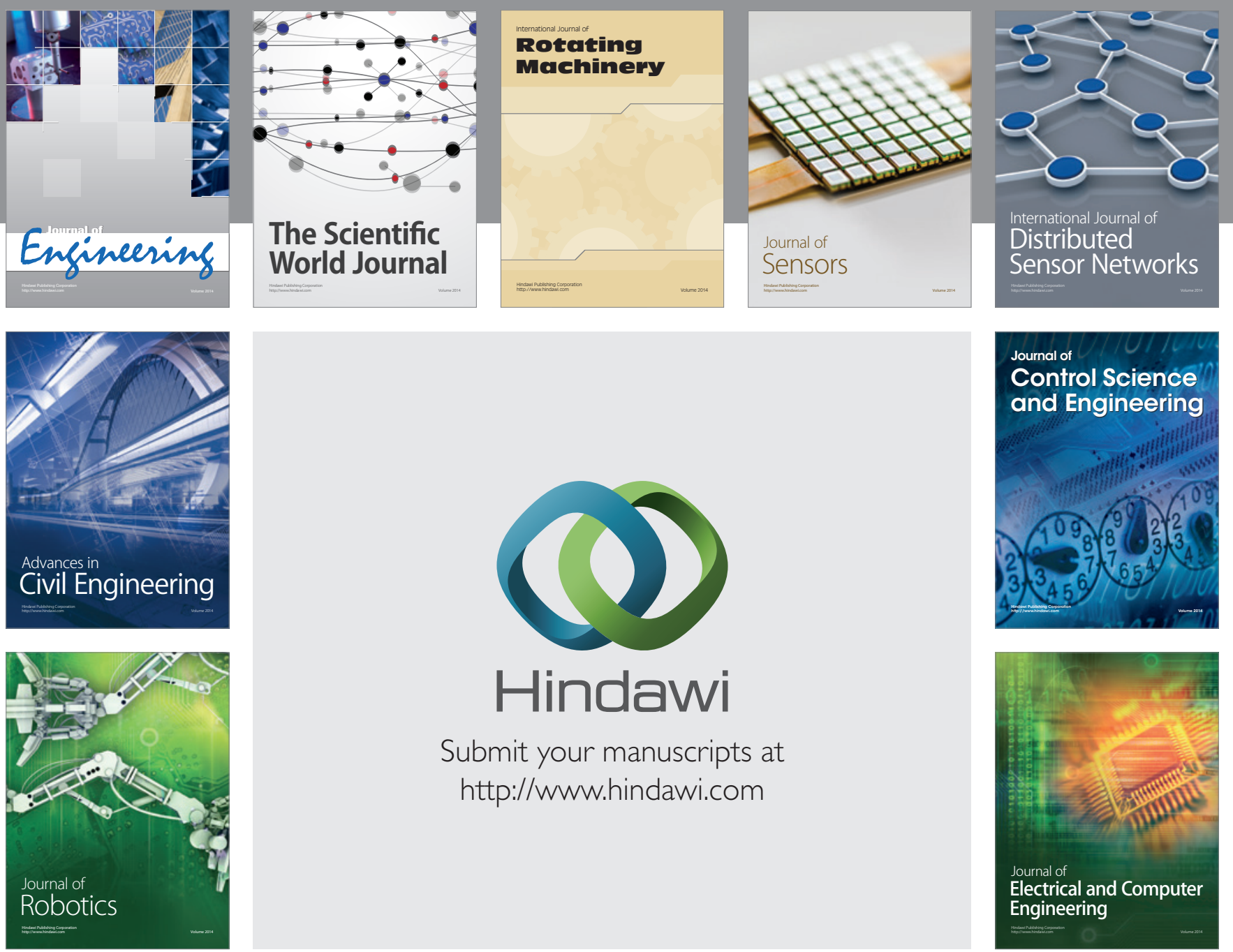

Submit your manuscripts at

http://www.hindawi.com
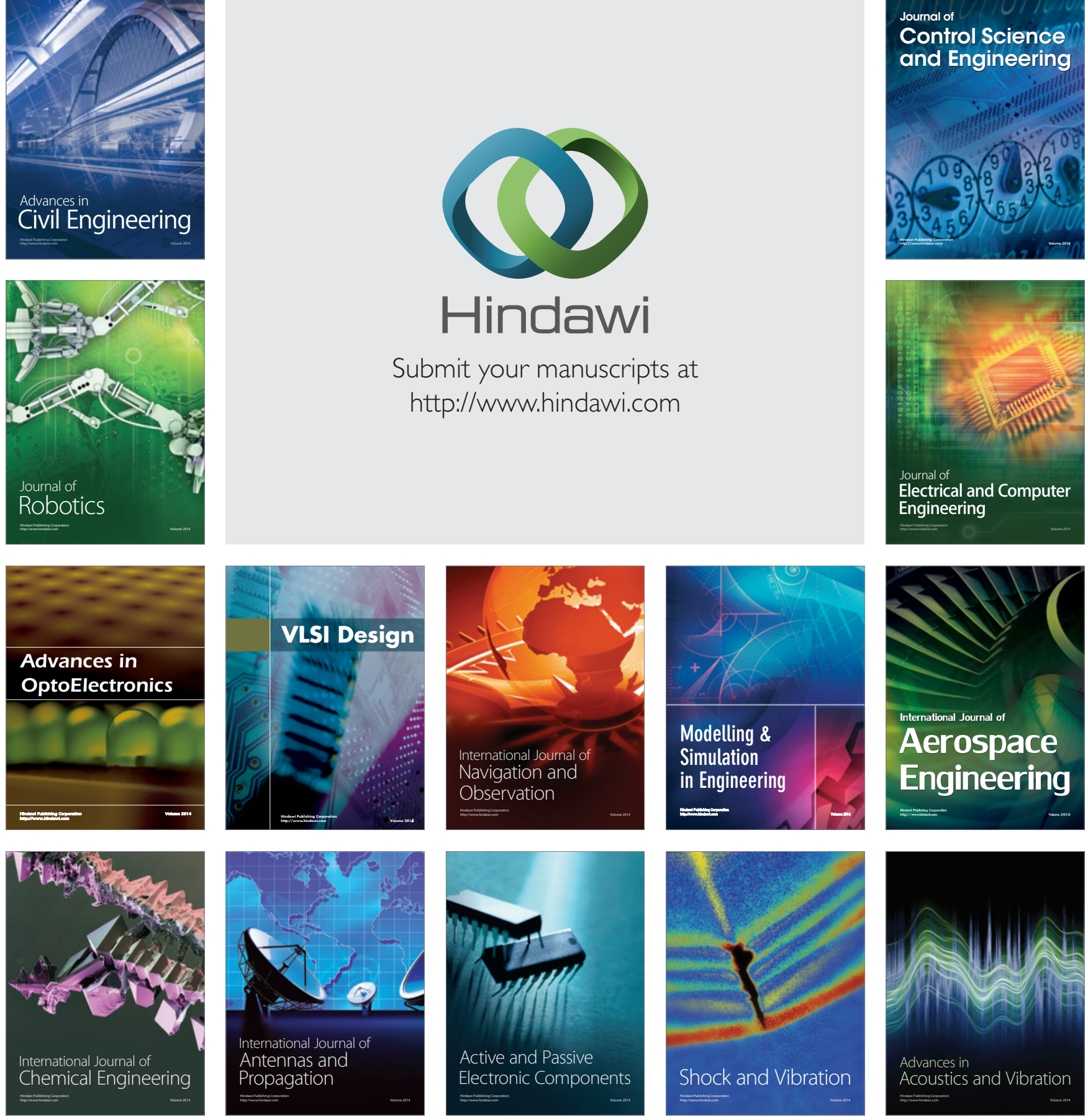